\title{
Verwendung der Reisinger'schen Hakenpincette bei der Cataractextraction.
}

\author{
Von \\ Professor Arlt.
}

Bei der Extraction mit dem Bogen- oder mit dem peripheren Linearschnitte wird darauf gerechnet, dass die linse nach Eröffnung der Kapsel aus dem Auge herausgedrückt werde, von selbst (durch den Druck, welchen die äusseren Augenmuskeln im Vereine mit dem M. orbieularis auf den Bulbus üben), oder unter Nachhilfe des Operateurs (mit dem Finger, mit Instrumenten).

Dieses Manöver setzt voraus, dass in der Zeit, in welcher der Druck auf den Bulbus wirkt, die Zonula und die Hyaloidea ihre normale Widerstandsfähigkeit besitzen und dass sie dabei nicht gewaltsam verletzt werden (durch übermässigen Druck, durch Zerrung, durch Instrumente).

Die gewaltsame Dehnung und Zerreissung kann schon beim Durchführen des Messers durch Druck der Klinge nach hinten, durch Zerren der zu durchschneidenden Gebilde oder durch hastige (schnalzende) Beendigung des Schnittes bewirkt werden; auch bei der Handhabung des Cystitoms (Fliete, Häkchen, Nadel) kann eine Verwundung der Zonula und eine Zerreissung derselben mittelst Ver- 
schiebung der Linse eintreten; aber am häufigsten dürfte die Ursache gewaltsamer Zonulatrennung und dadurch veranlassten vorzeitigen Glaskörperaustretens darin liegen, dass der Operateur, indem ex die in den vorausgeschickten Momenten begangenen Fehler (zu kleinen Hornhautschnitt, ungenügende oder nicht erfolgte Kapseleröffnung, Verschiebung der Linse) nicht erkennt, das Austreten der Linse durch forcirten Druck auf den Bulbus zu erzwinger strebt.

Auch ohne Verschulden bei dem Ausführen der Extraction kann es geschehen, dass Zonula und Hyaloidea einreissen, weil sie nicht die normale Widerstandsfähigkeit besitzen oder weil die Zonula theilweise oder ringsum defect ist. In solchen Fillen ist es Sache des Operateurs, diesen Zustand in vorhinein zu erkennen oder doch als wahrscheinlich vorauszusetzen, daher bei Zeiten darauf vorbereitet zu sein. Hierber gehören nicht nur die Fälle von Ectopia und Luxatio lentis (spontan, traumatisch), sondern auch Falle 'mit Sehlottern der Linse, Fälle mit Erweiterung des Ringes, welchen der Ciliarkörper beschreibt (namentlich bei hoehgradiger Kurzsichtigkeit mit allgemeiner Scleralausdehnung), Fäle mit Cataracta nigra, manche Fäle mit Kapsellinsenstaar (überreife Staare mit Sobrumpfong der verdickten Kapselpartie).

In allen diesen verschuldeten und unverschuldeten Fallen tritt gleich nach Beendigung des Hornbautschnittes oder nach Eröffnung der Kapsel, mitunter erst in dem Momente, in welohem man daran geht, den Staar austreten zu lassen (wenn der Kranke die Lider krampfhaft bewegt oder den Athem gewaltsam zurückhalt, besonders bei mehr flach liegenden Augen), Glaskörper in die Wunde und drängt die Linse ron der Wunde $a b ;$ die Linse kann dann mur durch ein Tractionsinstrument aus dem Auge befordert werden. Das Zurüeklassen der Linse unter solchen Umständen hat meistens, namentlich wenn bereits 
die Kapsel eröffnet ist, den gänzlichen Verlust des Auges zur Folge.

Zur Zeit der sogenannten Lappenextraction wurden in solehen Fallen zum Herausziehen der Linse nur der Davielsche Löffel oder das einfache spitzige Irishäkchen, beim Kapsellinsenstaar wohl auch eine Irispincette verwendet.*) Als dann Graefe daran ging, die lineare Extraction (Extr. mit linearem Schnitt nach Ed. Jaeger**) nicht bloss bei flussigen, weichen und geschrumpften Staaren anzuwenden,**) sondern auch bei Staaren mit hartem Kerne durch die Combination mit Iridectomie $\dagger$ ) möglich zu machen, wurden besondere Tractionsinstrumente, zunächst ein modificirter Davielscher Löffe? (ibid. pag. 163) in die Praxis eingeführt, anfangs für die einfache modificirte, bald darauf für die periphere Linearextraction. Den Typus der später angegebenen Tractionsinstrumente bildeten die 1860 von Waldau (Schuft) angegebenen und von Graefe $\dagger$ ) adoptirten Löffel (in 4 stufenweisen Grössen). Man konnte damit wohl die Linse leichter fassen und gefasst erhalten, aber durch den breiten und steil aufsteigenden Rand wurde schon beim Einführen des Loffels und noch mehr zu Ende der Traction die Herausdrängung einer beträchtlichen Menge von Glaskörper bewirkt; überdies waren die Wundränder besonders beim Eintreten der Linse in die Wunde sehr der Quetschung ausgesetzt. Diesem Uebelstande suchte Critehett dureh Weglassung der steilen Ränder zu beiden Seiten der Platte zu vermindern, und Ad. Weber reducirte sie dann auf ein Geringes durch seine Drahtschlinge, welche ron

*) Vergl. Arlt, Krankheiten des Auges, Prag 1854, II. B. pag. 286, 298, 300 and 314 .

**) Staar und Staaroperationen, Wien 1854.

***;) A. f. O. T. b. pag. 219 (1855).

t) Modificirte Linearextraction, A. f. O. V. a. pag. 158 (1859).

t?) A. f. O. VI. b. pag. 155 . 
dem Waldau'schen Löffel gewissermassen nur den Rand beibehielt; dieselbe hat wohl unter allen Tractionsinstrumententen die grösste Verbreitung gefunden.

An der hinteren Flache der Linse bis unter deren Centrum hinabgeführt, ohne viel Flüssigkeit zu verdrängen, and dann den harten Kern gegen die Cornea angedrückt erhaltend, kann dieses Instrument das Ausgleiten des Gefassten nach den Seiten wie nach der dem Wundcanale entgegengesetzten Richtung verhindern. Nachdem ich vor einigen Jahren diese Drahtsehlinge an der coneaven Seite and gegen die Oese zu mit feinen queren Riefen (Kerben) versehen liess, scheint mir dieselbe noch mehr Sicherheit gegen das Ausgleiten zu gewäbren, enthebt indess nicht der Nothwendigkeit, während der Traction continuirlich einen beträchttichen Druck gegen die Cornea einzuhalten und hierdurch namentlich bei Beendigung des Zuges Anstoss zu stärkerem Glaskörperverlust zu geben. Ich habe seit der Adoptirung der Graefe'schen peripheren Linearextraction als Tractionsinstrument, wenn ein solches zur Beendigung der gewobnlichen Extraction nöthig wurde, den Graefe'schen Loffel, später nur die Weber'sche Drahtschlinge angewendet und so manches Ange, welches sonst zu Grunde gegangen wäre, noch gut durehgebracht, aber: doch relativ viele der so operirten Augen bald durch suppurative, bald durch schleichende plastische Iridokyklitis verloren. Man ist, wenn einmal die Kapsel erüfinet wurde, nicht immer so glücklich, die Linse mit dem Loffel oder mit der Schlinge gänzlich herauszufördern, das Manöver ist selten ohne Druck und Quetsching moglich und man kann gar oft der Anzeige für richtigen Wrndschluss nicht genügeleisten. Demnach betrachte ich es immer als etwas Missliches, wenn ich in Fallen, in denen auf spontanen Austritt der Cataracta (durch blossen Druck) gerechnet werden konnte, zu einem Tractiunsinstru- 
mente greifen muss. Melius remedium anceps quam nullum.*)

Alle bisher üblichen Tractionsinstrumente bewirken die Herausbeförderung der Linse nicht durch blosses Anziehen, sondern nur unter stetem Andrücken der Linse gegen die Cornea. Sie ersetzen den Druck, welchen bei normalem Vorgange der Extraction der Glaskörper von hinten ausübt, durch eine Platte oder Schlinge. Soll die Linse beim Anziehen gegen die Wunde nicht ausgleiten, so muss der Druck gegen die Cornea um so stärker sein, je weniger das Instrument mit seinem vorderen Ende die Linse umgreift. In dieser Beziehung wären der WaldauGraefe'sche und der Critchett'sche Löffel dem Pagenstecher'schen und der Weber'schen Drahtschlinge rorzuziehen; aber sie verdrängen beim Einführen und beim Umgreifen der Linse um so mehr Flüssigkeit, je höher und steiler die Ränder der Platte emporstehen.

In jenen Fällen von Cataracta, in welchen auf Heraus beförderung der Linse durch Druck nicht gerechnet werden kann, ist die Eröffnung der Kapsel gar nicht möglich oder doch nicht rathsam. Die Eröffnung der Kapsel setzt normale Beschaffenheit und normale Befestigung derselben (an den Ciliarfortsätzen) voraus. In den oben angeführten Fällen dieser Kategorie ist es notwendig oder doch durch die Vorsicht geboten, das operative Eingreifen gleich in vorhinein so einzurichten, dass die Linse sammt der Kapsel herausgezogen werden lann und

*) Anm. Der Loffel von Pagenstecher (Klin. Beobachtungen 1866 pag. 24), zur Extraction der Linse sammt der uneroffneten Kapsel bestimmt, hat sehr dünne und kaum merklich nach innen umgebogene Ränder, ist $9 \mathrm{Mm}$. Iang, $6 \mathrm{Mm}$, breit und $1 \mathrm{Mm}$. tief; seine Höhlung entspricht ohngefähr der Krümmung der Linse. $\mathrm{Er}$ ist ans Silber angefertigt.

Zur Extraction einer dislocirten verkalkten Linse musate Graefe (A. f. O. II. a. pag. 195) einmal eine starke Pincette zu Hilfe nehmen.

v. Graefe's Archiv fïr Ophthalmologie, XXXI, 4. 
dass dabei möglichst wenig oder gar kein Glaskörper verm loren geht. Hier ist ein Fassinstrument unentbehrlich und verdient jenes den Vorzug, welches bei der nothigen Sicherheit für das Herausziehen am wenigsten Gefahr stärkeren Glaskorperverlustes bietet. Als solches betrachte ich das einfache spitzige Irishäkchen und in Rücksicht der grösseren Sicherheit das Doppelhäkchen, welches 1816 von Reisinger zur Pupillenbildung angegeben worden ist.*) Als zweokmässig kann ich (nach Erfahrung in einigen Fallen) folgenden. Vorgang empfehlen.

Eine Iridéctomie wird vorausgeschickt (ca. 6 Wochen) nach oben, wenn nicht besondere Gründe, z. B. Bctopie der Linse, für eine andere Stelle sprechen. Der Einstich wird nicht im Scleralborde, sondern nächst dem freien Rande des Limbus gemacht, mit einem Lanzenmesser, 5-6 Mm. lang und so steil, als es die Tiefe der Kammer gestattet. Es ist nicht nöthig, ein breites und tiefes (bis

*) Ein neues Verfahren, die Mastdarmfistel zu unterbinden and eine leichte und sichere Methode, künstliche Pupillen zu bilden, Augsburg 1816.

Die Abbildung der Reisinger"schen Hakenpincette findet sich anch in Blasius Akiurgie XVII 47 (nach Himly) und in Andreae Grundriss der Augenheilkunde, Leipzig 1846, I. B. pag. 345 mit Fig. 27.

Ioh besitze ein solches, walhrscheinlich aus der Zeit seiner Eiffindung stammendes Instrument und habe nur die nadelförmigen Häkchenträger etwas kürzer máchen und die Federkraft so weit vermindem lassen, dass die Häkehen nicht weiter als anf 4 Hillimeter auseinander weichen. (Siehe beistehende Abbildung). Werden die Branchea greschlossen gehalten, so bildet der vordere Theil ein einfaches Irishäkchen und kam ganz wie ein solches gehandhabt werden. Damit die platten Imenflächen der Häkchen und ibrer Träger: bein Schliessen genau an einander passen uxd sich nicht verschieben, steht bei 0 an der einen Branche ein Zapfen vor, welcher beim Schliessen in ein an der anderen Branche gegenitber" befindliches Loch eingreift. Das ganze könnte wohl etwas kiüzer gebaut werden, uameatlich auf Kosten des Schaftes (Griffes). 
Verwendung der Reisinger'schen Hakenpincette etc. 291

in den Ciliarrand reichendes) Colobom zu bilden. Iriseinklemmung ist sorgfältigst zu verhüten.

Die Verlegung des Einstiches an den Rand des durchsichtigen Bereiches der Cornea ist Reisinger's aus zwei Gründen angezeigt; erstens hindert pinette. dann die Narbe nicht die. Bildung eines Bindehautlappens bei der nachfolgenden Linearextraction, und zweites entsteht bei dieser Lage der Hornhautwunde nicht so leicht eine Einklemmung von Iris in den Wundwinkeln als bei Verlegung der Wunde in den Seleralbord. Aus letzterem Grunde ziehe ich jetzt auch bei Glaucom es vor, den Einstich nicht mehr so peripher wie früher zu machen. Der Streifen der Iris, welcher am Grunde des Coloboms sitzen bleibt, wird im Verlanfe einiger Wochen so schmal, dass er dann bei der Extraction weder das Einführen der Instrumente, noch das Austreten der Linse behindert.

Das Fassen des Sphineters behufs der Excision lässt sich in Fällen, in welchen derselbe keine Stütze an der Linse findet (bei wässriger Beschaffenheit des Glaskörpers, bei Luxation der Linse u. s. w.), nieht mit der Pincette, sondern nur mit einem spitzigen Irishäkehen bewerkstelligen.

Ein Colobom, welches zu der Zeit, wo die Extraction vorgenommen wird, die Breite von mindestens $3 \mathrm{Mm}$. erlangt hat, erleichtert bei dieser das Durchführen des Messers durch die Kammer, wenn diese an Tiefe zu wünschen übrig lässt, und gewährt nach der Extraction beinahe volle Sicherheit gegen Iriseinklemmung

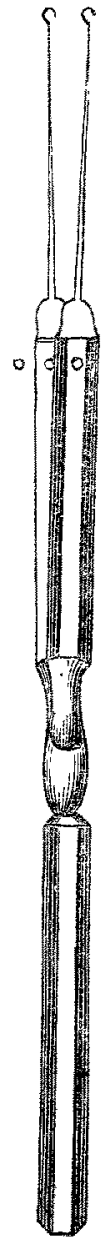
und cystoide Vernarbung.

Bei der Extraction (im buchstäblichen Sinne) - ich 
ziehe jetzt den pheripheren linearen Schnitt nach oben, falls ex zulässig ist, wegen der leichteren (sicherern) Nachbehandlung dem typischen Bogenschnitte vor - ist die Narkosis in so fern wünschenswerth, als man Grund hat, grosse Unrahe des Patienten wegen Aengstlichkeit, Ungelehrigkeit oder Ungebärdigkeit zu befürchten. Ziemlich verlässliche Anhaltspunkte zur Beurtheilung dieser Eigenschaften gewährt die Beobachtnng während nnd nach der vorausgeschickten Iridectomie. Bei richtiger Verwendung des Cocaïns, entsprechender Lagerung des Patienten und Vermeidung des Sperrelevateurs wird man in den meisten Fallen ohne Narkosis gut zum Ziel gelangen und seine Aufmerksamkeit ungetheilt den Vorgängen am Auge zuwenden können.

Wăhrend bei erhöhter (beinahe sitzender) Lage des Patienten der Kopf durch Jemanden festgehalten wird, fixirt dex Assistent das obere und, weṇn nöthig, anch das wntere Lid. Zur Ausfuhrung des Hornhantschnittes eignet sich ein verjüntes Beer'sches Messer besser, als das Graefe'sche, weil es gestattet, den Schnitt fast ohne Zerrung am Bulbus zu vollenden. Die Klinge hat die Form eines sehr spitrigen Dreieckes; die Spitze liegt in der Flucht des dünnen stumpfen Rückens, die Schneide läuft geradlinig zur Basis, welche nahezu $3 \mathrm{~cm}$. hinter der Spitze liegt and $2-3 \mathrm{Mm}$. breit ist. Mit einem. solchen Messer kann der Schnitt fast ohne Zerrung ausgefüht werden, weil man durch Vorschieben, nicht durch Druck auf die Schneide wirkt und Sagebewegungen fast ganz vermeiden kann. Man kann damit auch einen zur Deekung der Corneoseleralwunde sich eignenden Bindehautlappen bilden. Der Bulbus bann bis zur Beendigung dos Schnittes mit einer Pincette (ohne Sperrvorrichtung) rom Operateur fixirt werden.

Der Patient ist in vorhinein anzuweisen, dass er auf Geheiss, das Auge zu schliessen, dies sofort und nur so 
Verwendung der Reisinger'schen Hakenpincette etc. 293

thue, wie man es zum Schlafen macht, den Kopf nicht steif (in die Kissen gedrückt) zu halten, mit den Händen. sich nirgends anzuklammern und ruhig (wo möglich mit offenem Munde) fortzuathmen. Bei krampfhafter Spannung der. Muskeln des Rumpfes and der Extremitäten erfolgt auch eine solche Action der Muskeln am Auge, und bei längerem Zurückhalten des Athems entsteht Stauung in den Hohlvenen, im Innern des Bulbus und Steigerung des intraocularen Druckes.

Nach einer kurzen Pause, in welcher man Thränen, Blut und ausgetretenen Glaskörper abtupft, wird der Kranke angewiesen, das Auge zu öfnen, wie wenn er geradeaus sehen wollte, der Assistent fixirt das obere und - nach Anlegung der Fixationspincette durch den Operateur auch das untere Lid, und der Operateur führt nun das einfache oder, was vorzuziehen ist, das geschlossene Doppelhäkchen durch die Wunde über den Rand der Linse an deren hintere Fläche und bis gegen den entgegengesetzten Rand, bis über die Mitte der anzuhakenden Linse.

Das einfache spitzige Irishäkchen hat bei dieser Einführung durch die Wunde, das Colobom und die Zonula (falls diese intact ist), über den Linsenrand und durch den Glaskörper bis unter den hinteren $\mathrm{Pol}$ der Linse keinen nennenswerthen Widerstand zu überwinden und verdrängt auch kein erhebliches Quantum von Flüssigkeit; es bewirkt nur ein geringes Klaffen der Wunde. Wird es dann dureh eine Vierteldrehung um seine Achse mit der Spitze nach vorn gerichtet und hierauf durch eine leichte Hebelbewegung (von hinten nach vorn) in die Kapsel und Linse eingesenkt, so kann einfaches Anziehen gegen die Mitte der Wunde leicht genügen, das Gefasste längs der Descemeti ohne erheblichen Druck herauszusehleifen, und die Wundränder werden schliesslich kaum mehr aus einander 
gedrängt, als es das Volumen der Cataracta erheischt.*) Wenn jedoch der Schwerpunkt der linse beim Anziehen nicht stetig mit der Führungslinie zusammenfüllt, weicht die Linse seitlich ans und ist das Ausgleiten schwer zn vermeiden.

Diesem Mangel an Sicherheit kann nach meinex Ansicht, gestutzt auf einige Palle, leicht abgeholfen werden durch die Reisinger'sche Hakenpincette oder wie ich sie lieber nennen möchte, das doppelte Irishäkchen. Ieh liess, wie oben erwähnt, dieses Instrumten so einrichten, dass die beiden Häkchen, welche geschlossen nur ein Häkchen bilden, von selbst (duroh Tederkraft) nicht weiter als bis auf $4 \mathrm{Mm}$. von einander weichen. Wird dieses Instrument geschlossen bis unter den hinteren Pol der Linse (cirea $2 \mathrm{Mm}$.) hinabgefuhrt, mit der Spitze nach rorn gedreht, dann seiner Federkraft überlassen und durch eine leichte Hebelbewegung in die Kapsel und Linse eingeseukt, so kann das Gefasste unter gelindem, doch stetem Anlegen an die Descemeti leicht und sicher herausgezogen werden. Beim Durchtreten durch die Wunde kommt der Bogen, welchen jedes Hakchen bildet, nicht in Betracht, weil die Hükchen zur Seite der dicksten Stelle der Linse in diese eingesenkt sind, daher kein stärkeres Klaffen der Wunde bewirken, als es ter sagittale Linsendurchmesser erfordert.

* Vergl. AnIt, Krankheiter II. B. pag. 314 und Operationslehre in Graefe-Snemisch, III, B. pag. 277. 